Kenneth Silver

penultimate draft

\title{
Causal Exclusion and Ontic Vagueness
}

\begin{abstract}
The Causal Exclusion Problem is raised in many domains, including in the metaphysics of macroscopic objects. If there is a complete explanation of macroscopic effects in terms of the microscopic entities that compose macroscopic objects, then the efficacy of the macroscopic will be threatened with exclusion. I argue that we can avoid the problem if we accept that macroscopic objects are ontically vague. Then, it is indeterminate which collection of microscopic entities compose them, and so information about microscopic entities is insufficient to provide a complete explanation of certain properties of macroscopic objects. After outlining this solution, I consider several objections.
\end{abstract}

\section{An Exclusion Problem for Macroscopic Objects}

Consider the case of one billiard ball (Ball 1 ) striking another (Ball 2 ), causing the second to zoom off in the opposite direction. This is paradigmatic causation if anything is, but this is in tension with several other plausible claims. Each ball is composed of molecules. And there are many molecular interactions that occur during the striking. We also might take the movement of the balls to be determined by the movement of the molecules. From these ideas, it seems that we can give a complete explanation for the movement of Ball 2 in terms of the causal interactions of the molecules. Critically, this explanation does not mention Ball.

This would not be a problem if the billiard balls were identical with the collection of molecules that make them up. 1 Then, the causing of the movement of Ball 2 by Ball 1 could be identified with the causing of the jettisoning of the second collection of molecules (Molecules2) by the first (Molecules 1 ). However, many metaphysicians do think that billiard balls are distinct from the collections of molecules that compose them. The billiard balls seem to have properties not had by the collection of molecules composing them, after all, and the billiard balls may seem capable of losing or slowly replacing which molecules compose them.

The arguments for the distinct existence of billiard balls and ordinary objects like them are numerous, and the debate concerning them is heated. If, however, we think that billiard balls are distinct from the molecules that compose them, then we are left with two distinct explanations for the movement of Ball 2 in our example. One explanation for the movement of Ball 2 is that it is caused to move by being struck by Ball 1 . But a distinct explanation is that $\mathrm{Ball}_{2}$ is determined by the molecules that compose it, and those molecules are caused to move as they are by Molecules1. This is an example of a tension between what Jaegwon Kim calls 'horizontal' determination and 'vertical' determination,2 and this is one manifestation of the causal exclusion problem in the context of ordinary objects.

1 The billiard ball is identified with a collection of molecules, but how we understand this depends on what we think about composition. We may take our references to a singular billiard ball to refer to a plurality of distinct molecules (if we take composition to never occur), or we may think that there is a singular billiard ball that is identical with this plurality (if we take composition to be identity). We are not committed to a particular reductive thesis here. For the sake of ease, I will assume the latter reductive thesis moving forward, so objects are threatened with exclusion by the entities that compose them.

2 Attributing this to Jonathan Edwards, Kim introduces 'Edwards's dictum' as follows: 'There is a tension between 'vertical' determination and 'horizontal' causation. In fact, vertical determination excludes horizontal causation' [2005: 36]. Much of Kim's discussion of the exclusion problem is governed by this, that there is a competition for what determines a given higher-level effect, and that the supervenience base that vertically determines it wins. 
Kenneth Silver

penultimate draft

Typically, causal exclusion problems concern one physical effect's having two distinct sufficient causes (and so being causally overdetermined).3 However, we can see just as plainly that there is overdetermination in this case. If causation and composition are both determination relations, then the state of $\mathrm{Ball}_{2}$ is determined twice over, and so overdetermined. 4 When confronted with this overdetermination, we face pressure to either show how the two explanations are identical after all or somehow privilege the vertical explanation-robbing Ball 1 of its claim to efficacy.

In this instance, Molecules1 seem to do the causal work that we thought Ball 1 was doing. More generally, each time Ball seems to be a part of some causal explanation, that is excluded by a separate and more fundamental explanation at the molecular level. If all of the work of Ball is excluded, then this robs at least one natural reason for positing the existence of Ball: We will not need it to give complete explanations of macroscopic phenomena. Moreover, there's nothing particularly special about billiard balls. Any time that any composed object appears to cause something, that effect has a sufficient explanation in terms of the objects composing it. This erodes our motivation for thinking that any composed object exists distinctly from the objects that compose it, leaving us only with the existence of mereological simples (if any even exist).

Exclusion problems of this kind were first popularized in the philosophy of mind [Kim $2000,2005]$. There, the question is whether mental properties reduce to physical properties of the brain. However, exclusion problems have since been raised for many different kinds of entities. Merricks [2001] specifically argues that exclusion concerns can be raised for macroscopic objects, and we can see how the above case is an example. My focus will remain on the above case and with the exclusion problem in the context of macroscopic objects, although in the final section I will consider whether the answer I give applies in other contexts.

To answer the charge of exclusion, the non-reductionist has several options. She can accept that macroscopic objects do not cause (and so accept epiphenomenalism); she can find some way of arguing that micro and macro explanations do not compete; or she can point to macroscopic effects that do not have complete microscopic explanations. Each of these has been pursued, and it may even be that different instances of purported exclusion are better answered in different ways. Here, I want to explore a new version of the last kind of answer. If we take for granted that there is genuine metaphysical vagueness out in the world-that macroscopic objects are vague-then we can see that micro-level explanations cannot fully explain effects involving macroscopic objects.

In what follows, I will first discuss what it means to say that macroscopic objects are vague. Then, I will demonstrate how one can be drawn to the idea that vagueness could give a solution to the exclusion problem. The character of the solution will be quick, and ontic vagueness is controversial, so the rest of the paper will involve clarifying the position by way of considering objections. This will invite us to consider more closely how ontic vagueness leads to this solution, how much vagueness is involved, why vagueness is necessary, and how the solution relates to others.

\footnotetext{
3 Framing exclusion arguments this way allows us to use the idea that causal overdetermination is impossible (or rare), and it allows us to use a closure principle whereby physical effects have sufficient physical causes.

4 It is not as straightforward to show how this kind of overdetermination is problematic as it is in the case of direct causal overdetermination. For the latter, we can appeal to the intuition that effects almost never have two sufficient causes. Although this case is not direct causal overdetermination, it does involve two distinct explanations, and both explanations are at least in part causal. Exactly how to motivate why this is intolerable is an interesting question, but I will take it for granted that it is a problem here, as both Jaegwon Kim and Trenton Merricks frame cases like this as leading to an exclusion problem.
} 
Kenneth Silver

penultimate draft

\section{How Ontic Vagueness Solves the Problem}

Vagueness is discussed in many contexts, but I will say that a macroscopic object is vague just in case there is some item for which it is indeterminate whether that item is a part of the object. We typically discuss objects vague at their boundaries, where there are many small skin cells, hairs, etc. for which it is unclear whether or not they are a part of the object (for instance, the cat with many hairs slightly attached or just detached but that still haven't fallen off). As we will see, however, some objects might be vague throughout, with no smaller item that is determinately a part of them. 5

In response to these cases of purported vagueness, we could say that the vagueness of the objects is some fault of the inexactness of our language or concepts. So, the word 'cloud,' for instance, does not determine which water molecules in the vicinity will count as parts of it. Alternatively, we could argue that there is no vagueness; we just do not know the true extensions of our terms and concepts. In contrast to these approaches, the proponent of metaphysical or ontic vagueness is drawn to the idea that the objects themselves are vague. That is, when it is indeterminate whether some item is a part of the object, what we are saying is that it is metaphysically indeterminate; there is no fact of the matter concerning whether that item is a part of the object. Ontic vagueness is not a popular view in metaphysics, or even among those working on vagueness for that matter. Still, it does have proponents, and I believe that a sincere advantage of the view is that provides the resources to avoid the exclusion problem for macroscopic objects.

As macroscopic objects, even billiard balls are prime candidates for being metaphysically vague, at least at their boundaries. There are molecules along their perimeters for which it is vague whether they should be counted among the parts of those billiard balls. Since we understand vagueness in terms of indeterminacy, it seems appropriate to say that for a given molecule along the perimeter of a billiard ball, it may be indeterminate whether that particular molecule is a part of the ball. If it is indeterminate whether a certain molecule is a part of the ball, then it is indeterminate for a given collection of molecules whether that collection is the collection that composes the billiard ball.

Here is the crucial move. It seems appropriate to think that if it is indeterminate which candidate collection of molecules composes Ball2, then no particular collection of molecules determines Ball2. After all, no collection of molecules can fully explain exactly where $\mathrm{Ball}_{2}$ is or how fast the ball is moving after it's been hit by Ball. But if no collection of molecules can fully explain the movement of $\mathrm{Ball}_{2}$, then no collection of molecules can offer a competing explanation for what determines the movement of Ball2.

We will still say that the movement of Ball 2 is caused by it's being struck by Ball 1 , and the events involving Molecules2 are caused by events involving Molecules1. And we need not deny that things happening at the molecular level involving the breaking of bonds and the transferring of energy are in some way related to the colloquial causal explanation of billiard balls striking. However, because of ontic vagueness, it is indeterminate whether the molecules

5 Carmichael [2011:316] discusses a world with items dispersed in such a way that it is vague whether anything is composed out of them. In such a case, it is equally true for each of the dispersed items that it is indeterminate whether it in part composes some further item. This is a case of what he terms a 'homogenous case of borderline composition,' which is in contrast to the 'heterogeneous' cases with which we are more familiar, where objects

have certain determinate parts and then candidate indeterminate parts in the penumbra. It is hard to think of homogeneous cases in the actual world, as most of our cases involve vagueness at the boundary. Though, perhaps a particularly wispy cirrus cloud may qualify, as one reviewer suggests. 
that make up the plurality of Molecules2 actually compose Ball2. Critically, the same will be true of any distinct collection of molecules in the vicinity of Ball 2 (and so all candidates for composing Ball2). Although there will be a complete explanation for the movement of every collection of molecules in the vicinity of Ball2, none of these can amount to a complete explanation of the movement of Ball2. Missing will be a fact of the matter concerning which collection actually composes Ball2, because there is no such fact. So, no competing distinct lower-level/molecular explanation can be given for the movement of Ball2.

Although we see this solution in the context of billiard balls, the vagueness solution is meant to answer the exclusion challenge for all plausibly vague macroscopic objects, and I take it to provide a response to the argument for the exclusion of everyday objects given in Merricks [2001]. There, Merricks discusses the case of a baseball that goes through a window, arguing for the claim that 'If the baseball exists, it does not cause the shattering of the window' [56]. Crucially, he relies on the premise, 'The shattering of the window is caused by [the atoms of the baseball] acting in concert' [ibid.], and this is what I hope to call into question. 6 The atoms of the baseball can't cause the shattering of the window; they can at most cause the scattering of the atoms that only indeterminately compose the shattered window.

The character of this solution is fairly straightforward, so let's move on to consider objections. Doing so will help us clarify the role of vagueness, the commitments entailed, and the relation of this solution to others.

\section{Objection I: Vague Objects Are Vertically Determined After All}

Even if we assume that objects can be vague, this solution may seem engaged in a simple conflation of 'determinate' and 'determined'. Just because it's metaphysically indeterminate which collection of molecules composes the billiard ball, this does not mean that the billiard ball is not determined. To say that the billiard ball is not determined by the molecules gives the odd impression that the ball somehow floats free of the molecules, only traveling in the same direction as them as a matter of happenstance. But this is not the case. The billiard ball is related to certain molecules, although it may be indeterminate to which ones. It's reasonable to think that we can use this fact alone to somehow demonstrate that Balle is vertically determined by molecules. But how?

\subsection{Determined by All Candidate Parts}

Instead of trying to think about which exact collection of molecules composes Ball2, perhaps we should think about the expanded collection that includes all of the molecules that are even a candidate for composing it. Although it may be indeterminate on which smaller collection of molecules Ball 2 supervenes (or the smallest collection on which it supervenes), it will surely supervene on this larger collection of molecules. There is no change in the properties of $\mathrm{Ball}_{2}$

6 Merricks claims that denying this premise entails emergentism, as the baseball must cause something that 'its parts, working in concert, do not' [62], and Merricks thinks that any such causation over-and-above something's parts entails some emergent property. This may be, and I will return to this in fn.9. However, vagueness alone does not entail emergence strictly given what Merricks says. The baseball does not necessarily cause anything that its parts, working in concert, do not. It may be perfectly acceptable to say that the parts of the baseball cause the window to shatter. But, we still will not have said that a particular collection of atoms is capable of causing the window to shatter. After all, it is indeterminate which atoms are parts of the baseball. 
Kenneth Silver

penultimate draft

without some change in the properties of this larger collection of molecules, and so Ball 2 seems determined by all of the molecules in this larger set.

Further, the properties of this larger collection of molecules may be causally explained by properties of the molecules in the largest supervenience base for Ball. So, even if there is no determinate set of molecules that plays a role in a competing explanation for the movement of $\mathrm{Ball}_{2}$, this does justice to the thought that the movement of Ball 2 is fully causally explained by molecules. Ball 1 is not causing anything not ultimately caused by some molecules.

To be sure, something is determined by the properties of this larger collection of candidate parts of Ball 2 , but is it really true that the movement of Ball 2 is determined by that collection? And, is it determined in a way that should make us fear exclusion? Consider the speed of Ball. Some of the molecules that are candidates for composing it are moving at some exact speed, while other candidate molecules are moving at a different exact speed. We can say that Ball 2 does not move slower than the slowest of those candidate molecules, nor does it move faster than the fastest. Still, this is insufficient to determine the speed of Ballz.

When we think of what it means for the molecules to determine the properties of the ball in a way that should worry us, we are likely thinking of some kind of metaphysical necessitation. The properties of the molecules guarantee the properties of the ball, or perhaps we think that they even generate the properties of the ball. But, when thinking of the larger supervenience base, the collection of molecules is not able to do either of these things. The molecules at best guarantee that the speed of Ball 2 is within a certain range. But this is not in competition with the direct causal explanation of the speed of Ball 2 (its being struck by Balli). It doesn't guarantee that the speed of Ball 2 is what it is, only that it is within some range. Further, the larger collection of molecules seems unable to generate the speed of the ball.7

If it's right to say Ball 2 gets its speed from the speed of its parts, then the speed will be vague, because it is indeterminate which molecules are among the parts of it. So, even the larger collection of candidate parts is incapable of determining the speed of Ball 2 in a way that threatens exclusion.

\subsection{Determined by Determinate Parts}

Instead of thinking about all of the candidate parts of the ball and what they determine, perhaps an opponent might turn to obvious parts of the billiard ball, molecules in the centre of it, and think about what they determine. Although it is indeterminate which collection of molecules composes the billiard ball, this is not to say that there are not many molecules that are determinately part of the ball. If these determinate parts of the ball have some specific speed and location, we might think that this would be sufficient to vertically determine the corresponding features of the ball.

Of course, this objection can only work if there are determinate parts, but we already acknowledged the possibility of cases of vague macroscopic objects without any determinate parts (fn.5). Assuming that exclusion problems can be raised for these entities as well, then an appeal to vagueness to solve them cannot be undermined by appealing to any determinate parts.

7 If the speed of Ball2 is vague, then there is no fact of its exact speed to be determined. Then, it seems that the molecules determine all of the facts to be determined, and there's nothing for Ball 1 to determine. However, it's a fact that Ball2 has a vague speed; no set of molecules is in a position to explain this fact; but the vague speed of Ball 1 as it collides with it is in a position to explain it. 
Kenneth Silver

penultimate draft

Even in the case of Ball2, though, the speed of a determinate proper part still cannot give the speed of the whole object. It merely gives you the speed of that part. If we take the speed of the whole to be a function of the speed of its parts, then even if we know that certain molecules are determinately parts (and we know their speeds) this would not suffice to give us the speed of the whole. Thinking again in terms of guaranteeing and generating, the speed of a determinate part does not guarantee the speed of the whole, since the average of the parts could be quite different, and it can at best only be partly responsible for generating the speed of the whole. As long as it is indeterminate which other molecules are the other parts of the ball, the speed of the ball will remain vague.

\subsection{Determined at Every Precisification}

As a final objection to my claim that the movement of Ball 2 is not vertically determined, an opponent might say that my use of vagueness for the solution cannot be supported by the views of metaphysical vagueness that have been given. Since few philosophers to start with accept the possibility of metaphysical vagueness, it better be that this solution to the exclusion problem is at least available to those that do. However, when one considers the more popular modal views of vagueness given in Akiba [2004], Williams [2008], Barnes [2010], or Barnes and Williams [2011], one might worry that they could not accept it.

Modal views of vagueness apply the common idea from linguistic views of vagueness that an instance of vagueness can be understood in terms of different precisifications, but instead of thinking of many ways that one might sharpen or make precise a concept, we should think about all of the different precise ways the world could be. So, if it is vague which molecules are parts of our billiard ball, then this will be a matter of the variety of ways that the ball could be precise (how some ways of drawing up the ball will include a certain edge molecule and some of them will not). Once we have these precisifications, the differences between the major views come out in how we should think of them. Akiba [2004] will say they provide something analogous to a modal or temporal dimension for objects, whereas Barnes and Williams will both say that the precisifications are genuinely modal and variously compose the object in different possible worlds. Williams [2008] says that in cases of vagueness more than one such possible world corresponds to reality, whereas Barnes [2010] claims that it is indeterminate which possible world is actualized.

The differences between these views is important and subtle, but an opponent can object to my solution given only what they have in common. They each begin by looking at the different precisifications, which are themselves non-vague objects. Then, the indeterminacy comes from how to think about them all together. But if this is right - if we are really talking about many precise, non-vague objects—one might think that the vague object is vertically determined after all, as each of the precisifications is determined.

Each precisification of the billiard ball is composed of some exact collection of molecules. So each precisification supervenes on and is determined by those molecules. Although we may say that the speed of the ball is vague, the speed of each precisification will not be vague-it's given by the speed of the molecules it determinately has as parts. But if each precisification of the ball is vertically determined by the molecules that determinately compose it, then how could it be that the ball itself evades vertical determination? It may be vague which way the world is, but every precise way that the world could be is such that the ball would be determined.

To make the problem seem even more damning, we can notice that on Barnes' view the vague object is even taken to be one of the precisifications-it's just unsettled which one 
it is.8 So, if all of the precisifications are vertically determined, and the vague object is one of the precisifications, then the vague object will be determined as well. It would be unsettled how it was determined, but it would still be determined.

In response, I admit that I am tempted to simply jettison these accounts of vagueness. Before saying more about this, though, I will show how someone could respond even while maintaining a modal view of ontic vagueness. Although each of the precisifications of Ball 2 can determine each of the speeds of the object they compose, it is not determined which composed object is Ball2. So, even if the speed of the ball is determined because the speed of each of the precisifications is determined, the speed of the ball is vague, and this is not determined by any or all of the precisifications. 9

It is at least a fact that the movement of Ball 2 is vague, and this is a fact that merely the molecules will be unable to explain. If Ball 2 is fully vertically determined, then Ball 1 may not count as part of the cause of Ball2's movement; nevertheless, we may still need to mention Ball 1 in a satisfying causal explanation of Ball2's movement.10 Ball 2 moves with a vague speed because it is struck by Ball. If reference to Ball 1 is still needed for causal explanations, then this may countenance accepting it in our ontology after all.

So, the resources are available for maintaining this solution to the exclusion problem even on these modal accounts. I should say, though, that I do not accept these accounts. The accounts of Barnes and Williams are sophisticated and well-motivated, and they can avoid many of the problems typically given for those who like ontic vagueness. Nevertheless, I do not think that the ball's being vague is a matter of different collections of molecules successfully composing it in different possible worlds and there being no fact of the matter as to which of those worlds is actual. This view hides indeterminacy between possible worlds, which at least Williams does not take to exist concretely [2008: 148]. It should be no surprise that we have a harder time using ontic vagueness to solve the causal exclusion problem on these views, because ontic vagueness is not even to be found within the actual world on these views.

Instead, if a composed object is ontically vague, I think we should just be ready to say that there is no fact of the matter for certain molecules as to whether those molecules are part it. Composition is a relation that may obtain or fail to obtain, but it may also be that for certain objects there simply is no fact of the matter as to whether composition obtains. This kind of view is less common among those who maintain ontic vagueness, but it does seem allowed for at least by Parsons [2000]. Parsons is explicitly concerned with indeterminacy rather than vagueness [28-29], and the indeterminacy primarily concerns states of affairs. Still, this is a view on which indeterminacy is in terms of there being no fact of the matter.

This is not the place to develop such an account of ontic vagueness. And any such account will have to answer all of the many hard objections that have been levied against ontic vagueness. Still, it strikes me as a plausible explication of a natural idea. On this idea, to say

8 This may be putting her view in a way that is unfair to both of us. It is more accurate to say that the precisifications pick out possible worlds, and it is unsettled which one is actualized. This at least makes it less clear that Ball2 would be strictly identified with one of its precisifications.

9 This may compel us to say that being vague is a property of macroscopic objects, and this property is not vertically determined by any microscopic entities. I cannot see why proponents of the modal views would not accept this. If so, then this may involve accepting emergentism after all.

10 This involves appealing to a difference between the causation relation and the relation of causal explanation. Davidson [1967], Lewis [1986], and Beebee [2004] take there to be a difference, where certain items (such as facts) may feature in causal explanations even if they do not count among the causes of the effect. For some such as Mellor [1995], however, causal explanations between facts are the central causal claims. 
Kenneth Silver

penultimate draft

that something is vague is to suggest that there is no fact in existence to answer to the issue at hand. Further, the missing fact is critical to why no set of molecules in our case can offer a satisfying complete explanation to compete with Ball.

\section{Objection II: This Entails Too Much Vagueness}

Philosophers regularly object to ontic vagueness in objects on the basis that it arguably leads to accepting too much vagueness or other, more problematic, kinds of vagueness (such as vagueness in composition or existence). This is not the place to defend ontic vagueness against this more general charge (and it is a problem for anyone who accepts ontic vagueness); however, we might think that the solution to the exclusion problem that I have given here incurs more vagueness than is normally accepted by proponents of ontic vagueness.

My solution requires that there are vague macroscopic objects. So, for certain microscopic objects there will be no fact of the matter as to whether those objects are parts of the macroscopic object. Does it follow from this that the relation of composition itself is vague? This does seem to be the textbook case of vague composition, since it will be vague which microscopic entities compose the macroscopic ones. Nevertheless, I do not think we are forced to say that the relation of composition vaguely obtains. I will put this issue to the side, however, since few authors have given objections to vague composition that go beyond objections to vague objects themselves.

It is sometimes argued that any ontic vagueness leads straightaway to vague existence, and that vague existence is problematic. I want to say first that I am not convinced that vague objects or vagueness in composition implies vagueness in existence or that it would even be a problem if it did. Hawley [2002] and Smith [2005] both take compositional vagueness to entail existential vagueness, and they go on to say why existential vagueness should be acceptable. Alternatively, Donnelly [2009] argues that compositional or mereological vagueness does not entail existential vagueness. Without settling this debate, I just want to recognize that proponents of any ontic vagueness will likely accept the vagueness of objects required to answer the exclusion problem.

What is unique to my discussion of ontic vagueness is that I am concerned with causation, and so we might worry that I am committed to vague causation. There has been very little discussion of vague causation, and we might take this to be a special problem for my view. However, we should note three things.

First, in so far as vague causation is a problem for me, it is likely a problem for any proponent of ontic vagueness. If events with vague objects will be derivatively vague, and if causation between such events is vague, then this is a result to which anyone who accepts vague objects will be committed. So, although there has been little discussion of it, it is not a new problem.

Second, it's not obvious why it must be a problem in the first place. How big of a problem we take it to be will likely depend on our antecedent view of the relation of causation itself. If we think causation is not a relation out in the world or that it is simply something to be analysed in terms of counterfactuals, then I don't see any motivation for taking vague causation to be problematic. (Perhaps this would just mean that on some precisifications there was counterfactual dependence while not on others.) It might seem more problematic if we take causation to be a force out in the world, or as some kind of diachronic law governed 
Kenneth Silver

penultimate draft

necessitation. Still, exactly why it is problematic for causation to be vague would have to be spelled out on a given account of causation.

Finally, it's not even obvious that the relation of causation itself would be vague. Even if it were indeterminate whether one thing was causing another, this may be explained by vagueness of the relata, not the relation. Vagueness in the causation relation itself, and such that it would be problematic, would require further argument.

\section{Objection III: Ontic Vagueness is Not Necessary to Solve the Problem}

Even if it does not entail more vagueness, accepting ontic vagueness is extremely controversial. The position is so unpopular that we might cast about for any other solution to the exclusion problem before considering it. And if one can be found, this would show us that ontic vagueness is not necessary to solve the exclusion problem.

This concern will not be very compelling if you antecedently like ontic vagueness (as I do). Ontic vagueness can be both intuitive and can provide satisfying answers to a bevy of problems in metaphysics (sorities arguments, the problem of the many, the causal exclusion problem). It would be extreme to accept ontic vagueness merely to solve the exclusion problem, but if we already found ontic vagueness compelling, then we should expect to use it in this way for the exclusion problem. For proponents of ontic vagueness, it would be odd to confront the exclusion problem and not use the fact that macroscopic objects are vague.

There is another form of this worry however. Even if we think that objects in the actual world are vague, we may not think that these objects are necessarily vague. There could be possible worlds in which there is no ontic vagueness. Further, we may think that there could be composed objects in these non-vague worlds. We would again face the causal exclusion problem for the objects in these worlds, but we will not be able to advert to their vagueness. If there is some solution that we could give for objects in these non-vague worlds, then it would seem that we should just accept that solution in the actual world as well.

Let's grant that ontic vagueness is contingent. Let's also grant for the moment that composition can occur in some of these worlds. We do not face an exclusion problem in these worlds, however, because the composed objects are not distinct from the objects that compose them. The exclusion challenge is only raised when we want to reify the existence of composed objects as distinct from that which composes them, but my intuitions about the facts of composition depend on facts about ontic vagueness.

As mentioned above, ontic vagueness can be used to help resolve a number of puzzles of composition, but a proponent of ontic vagueness such as myself thinks that it is necessary. Without ontic vagueness, we might be convinced by the Problem of the Many to say that mereological simples never compose objects. Or we might instead be compelled to accept a plenitudinous ontology where every collection of objects composes some further object. If simples never compose, then there will be no exclusion problem; and if we are convinced that simples always compose, then we may forfeit the intuition that playing a role in causal explanations is an important reason for positing an object's existence. Ontic vagueness is sometimes taken to be the price we must pay in order to have a theory of restricted composition (see Sider [2001: 123-4], Merricks [2005], Barnes [2007]), so we cannot just assume that there will be a causal exclusion problem in worlds without it.

Even if the actual world were shown to have no ontic vagueness, this would only convince me that the facts of composition must be different than I had supposed. Upon being 
shown precisely how some object is determinately made up by parts, it would become clear how the effects of that object could be understood in terms of the effects of those particular parts. The more transparent the connection between the composed object and its parts, the harder it would be to even conceive of the composed object as distinct. Demonstrating nonvagueness undermines the will to argue for the non-reductive view of composed objects, because the view is motivated by the opaque and indeterminate nature of the relationship between the micro and the macroscopic.

\section{Objection IV: Vertical Determination Is Necessary Anyway}

I claim that the movement of Ball 2 is caused by being struck by Ball 1 directly; however, we might find this kind of direct causation mysterious. Given the relationship between billiard balls and molecules, we might think that Ball 1 causes the movement of Ball 2 by causing the movement of Molecules2. Indeed, some in the literature have argued that higher-level entities like macroscopic events can only cause by so-called 'downwardly causing' a difference in the supervenience base of their higher-level effects. Kim [2000: 42; 2005: 39-40] directly endorses this for causation between mental events.

This is a problem for me, because I have tried to show how the vagueness of $\mathrm{Ball}_{2}$ undermines the ability of any collection of molecules to vertically determine it. But if Ball 1 can only count as causing Ball 2 to move via downward causation, then undermining the vertical determination of Ball 2 will not establish the causal role of Ball. To respond, I will of course deny that downward causation is necessary in this case. To see why, let's consider why Kim takes it to be required.

First, Kim is convinced that downward causation will be required because he is already convinced that a higher-level effect's supervenience base vertically determines it. If we were convinced that the properties of some event were entirely determined by the properties of a lower-level event on which it supervened, then we would also think that the only way to influence the higher-level event would be to affect the lower-level one.11 However, since I have argued precisely against the effect's being upwardly determined, we no longer need to say this. If the effect is not fully determined by its supervenience base, this opens up space to say that it is determined and directly caused by the higher-level cause.

Second, Kim takes it to be mysterious how one higher-level property could directly cause another without causing the properties on which it supervenes. As an example, he says, 'To relieve a headache, you take aspirin: that is, you causally intervene in the brain process on which the headache supervenes. That's the only way we can do anything about headaches' [2000: 42].

This makes downward causation in certain cases seem necessary, but this kind of concern is hard to take seriously in the case of billiard balls. Causation between billiard balls is paradigmatic, blue-blooded causation, as regular and un-mysterious as possible. Recognizing the causation between ordinary macroscopic entities may well be how we come about the concept in the first place. So, it would be ad hoc to claim that macroscopic entities can only

11 Related here is Kim's endorsement of what he calls the 'causal inheritance principle', which is roughly the idea that if a property is realized by some physical base, then the causal powers of that property are identical to those of its physical base [Kim 1992:18]. There is precedent for rejecting the principle outright [e.g. Pereboom 2002, Gibbons 2006], but I do not discuss this more at length because if there is no physical base of molecules to which the ball bears a determinate relation, then the antecedent of the principle is not satisfied anyway. 
Kenneth Silver

penultimate draft

cause by downwardly causing. Taking this reasoning to its natural conclusion, we would have to say that causation ultimately is only between the entities of fundamental physics [Block 2003], but causation at that level may work very differently from how we understand it.

By claiming that one billiard ball causes the movement of another without needing to downwardly cause, this puts my view in league with a certain class of solutions given to the causal exclusion problem that sometimes go under the heading of 'intralevelism'. Thomasson [1998] and Gibbons [2006], for instance, both take on a structured ontology in which nature is ordered causally into levels (chemistry, biology, the psychological, etc.) and events in each level only cause other events in the same level. These solutions also avoid exclusion in cases of downward causation, and they each have their own way of answering Kim's tension between horizontal and vertical determination.12

These views are attractive, but they can be hard to accept if we take seriously Kim's claim that the lower-level entities really necessitate the higher-level effects [2000:39-40]. As Buckareff [2011: 412-3] points out, it's unclear how causation and upward determination can coexist without leading to overdetermination if we accept both of them as generative notions.13

The best way to accept that the horizontal cause of the higher-level effect beats out the vertical determiner is if we have reason to believe that the vertical determiner is insufficient or incapable of necessitating the higher-level effect after all, and this is just what I have claimed. No group of molecules can necessitate the movement of $\mathrm{Ball}_{2}$, because $\mathrm{Ball}_{2}$ is vague and so not determinately related to any group of molecules.14

\section{Conclusion: The Scope of the Ontic Vagueness Solution}

Typically, solutions are heralded as the solution to the Causal Exclusion Problem. But my solution was built to handle the case of billiard balls. So, to conclude I want to speak to the scope of my solution, and to say why we shouldn't hold out for a more universal one.

We should not expect there to be one solution for all exclusion problems, because everybody should want some exclusion arguments to go through. Consider ghosts. One way we argue against their existence is by showing how everything they purport to cause already has a

complete explanation in terms of physical causes. A solution to an exclusion problem will be too general, then, if it undermines this argument.

Even of those exclusion problems we want to solve, we should not hold out for a general solution, as how a non-reductionist argues often depends on the relationship that the item bears to its purported excluder. Vague objects stand in a special indeterminate relationship with their parts, and it's in virtue of the nature of this relationship that they are not excluded. But other entities facing exclusion stand in other relationships with their purported excluders.

12 For Thomasson, there's no tension, as causation and upward determination are different and non-competing determination relations. Gibbons claims that if there is a tension then surely horizontal determination wins.

13 One way to secure the generative yet non-competitive nature of horizontal and vertical determination would be to fall back on answers to the exclusion problem that rely on the close connection between the excluded and the excluder. Such answers may claim there is no overdetermination [Bennett 2003, 2008] or that the overdetermination is acceptable [Paul 2007]. These may be successful ways of answering the causal exclusion problem in certain contexts, but they are not necessary when the entity is vague.

14 The cases for which the vagueness solution is viable do not require (and perhaps must not involve) downward causation. However, my solution is compatible with the possibility of downward causation elsewhere. 
For example, there is a question about whether statues are distinct from the lumps of clay that constitute them.15 Someone that takes them to be identical could argue that the causal role of a statue is excluded by the lump that constitutes it. A non-reductionist must answer this challenge, but there is no indeterminate relationship between the statue or the lump. The statue is not even composed of the lump. Both objects might be composed of molecules, but this will not tell us whether the statue is excluded by the lump. If the statue is not to be excluded, then this will be borne out by the nature of the relation that they stand to one another, the constitution relation. (And see Pereboom [2002, 2011] and Paul [2007] for solutions appealing to this relation.)

So, the vagueness solution does not need to apply everywhere that there is an exclusion challenge. Vagueness is not a panacea for exclusion. The vagueness solution is appropriate, however, in cases in which objects are threatened with exclusion by their parts. That there is no fact of the matter as to which items are among their parts is exactly what we should use to justify the explanatory force of the object's existence.

Acknowledgements: I would like to thank Shieva Kleinschmidt for encouragement, Rebekah Rice for commentary, and a colloquium audience at the 2018 Pacific APA meeting. Thanks also to Dan Pallies for helpful discussion.

\section{References}

Akiba, K. 2004. Vagueness in the World, Noûs 38/3: 407-29.

Barnes, E. 2007. Vagueness and Arbitrariness: Merricks on Composition, Mind 115/461: 105113.

Barnes, E. 2010. Ontic Vagueness: A Guide for the Perplexed, Noûs 44/4: 607-27.

Barnes, E. and Williams, J. R. G. 2011. A Theory of Metaphysical Indeterminacy, in Oxford Studies in Metaphysics (Vol. 6), ed. Karen Bennett and Dean W. Zimmerman, Oxford University Press: 103-48.

Beebee, H. 2004. Causing and Nothingness, in Causation and Counterfactuals, ed. John Collins, Ned Hall, and L. A. Paul, Cambridge, MA: MIT Press: 291-308.

Bennett, K. 2003. Why the Exclusion Problem Seems Intractable, and How, Just Maybe, to Tract It, Nô̂s 37/3: 471-97.

Bennett, K. 2008. Exclusion Again, in Being Reduced: New Essays on Reductive Explanation and Special Science Causation, ed. Jesper Kallestrup and Jakob Hohwy, Oxford: Oxford University Press: 280-305.

Block, N. 2003. Do Causal Powers Drain Away?, Philosophy and Phenomenological Research 67/1: 133-50.

Buckareff, A. 2011. Intralevel Mental Causation, Frontiers of Philosophy in China 6/3: 402-25.

Carmichael, C. 2011. Vague Composition Without Vague Existence, Nồs 45/2: 315-27.

Davidson, D. 1967. Causal Relations, Journal of Philosophy 64/21: 691-703.

15 The question can be raised for artifacts generally. Billiard balls are also artifacts, which invites confusion, but I have been concerned with whether billiard balls qua macroscopic objects are excluded by microscopic objects. Another question is whether billiard balls qua artifacts are excluded by the acrylic balls that constitute them. 
Kenneth Silver

penultimate draft

Donnelly, M. 2009. Mereological Vagueness and Existential Vagueness, Synthese 168: 53-79.

Gibbons, J. 2006. Mental Causation Without Downward Causation, Philosophical Review 115/1: $79-103$.

Hawley, K. 2002. Vagueness and Existence, Proceedings of the Aristotelian Society 102/1: 125-40.

Kim, J. 1992. Multiple Realizability and the Metaphysics of Reduction, Philosophy and Phenomenological Research 52/1: 1-26.

Kim, J. 2000. Mind in a Physical World: An Essay on the Mind-Body Problem and Mental Causation, first MIT Press paperback edition, Cambridge, MA: MIT Press.

Kim, J. 2005. Physicalism, or Something Near Enough, Princeton: Princeton University Press.

Mellor, D. 1995. The Facts of Causation, London: Routledge.

Lewis, D. 1986. Postscripts to 'Causation', in Philosophical Papers (Vol.2), Oxford: Oxford University Press: 172-213.

Merricks, T. 2001. Objects and Persons, Oxford: Oxford University Press.

Merricks, T. 2005. Vagueness and Composition, Mind 114/455: 615-37.

Parsons, T. 2000. Indeterminate Identity: Metaphysics and Semantics, Oxford: Oxford University Press.

Paul, L. A. 2007. Constitutive Overdetermination, in Topics in Contemporary Philosophy: Volume 4: Causation and Explanation, ed. Joseph Keim Campbell, Michael O’Rourke, and Harry Silverstein, Cambridge, MA: MIT Press: 265-90.

Pereboom, D. 2002. Robust Non-Reductive Materialism, Journal of Philosophy 99/10: 499-531.

Pereboom, D. 2011. Consciousness and the Prospects of Physicalism, Oxford: Oxford Press.

Sider, T. 2001. Four-Dimensionalism, Oxford: Oxford University Press.

Smith, N. J. J. 2005. A Plea for Things that aren't Quite All There: Or, is There a Problem about Vague Composition and Vague Existence?, Journal of Philosophy 102/ 8: 381-421.

Thomasson, A. 1998. A Nonreductive Solution to Mental Causation, Philosophical Studies 89: 181-95.

Williams, J. R. G. 2008. Multiple Actualities and Ontically Vague Identity, The Philosophical Quarterly 58/230: 134-54. 